\title{
Molecular signatures of phenotypic and behavioral plasticity in bees
}

\author{
Klaus HARTFELDER \\ Faculdade de Medicina de Ribeirão Preto, Universidade de São Paulo, Avenida Bandeirantes 3900, Ribeirão Preto, SP \\ 14049-900, Brazil
}

This issue of Apidologie brings a special series of review and original research articles on molecular signatures underlying gene expression patterns related to the amazing diversity and versatility of bees and their social structure. The series is divided into two major sections; the first one brings insights from a non-social and an incipiently social species, while the subsequent three articles address questions of developmental gene expression in specific tissues of the honey bee, Apis mellifera.

The first article, provided by Maria Cristina Arias and members of her group, addresses the phenomenon of bivoltinism/diapause in the nonsocial tropical bee Tetrapedia diversipes. Bivoltinism associated with diapause in the second offspring generation has been proposed as a factor favoring the evolution of insect sociality in temperate climates. Here, this hypothesis was investigated from a transcriptomics perspective, comparing larvae from the first generation (direct developers) with those of the second one (larvae enter diapause). A series of genes related to oxidative metabolism and immune responses, as well as certain long noncoding RNAs were identified as differentially expressed among the two generations. The molecular markers and genomic information now available make this an interesting species to study developmental questions related to social evolution in tropical bees.

The second article, written by Wyatt Shell and Sandra Rehan, reviews the sociogenomic background related to incipient sociality in small carpenter bees and sweat bees. Well known for their behavioral and phenotypic plasticity, these bees are now receiving more and more attention in molecular studies, and this requires that the respective results are interpreted within a phylogenetic framework. Genetics, genomics, and transcriptomic insights are presented in the context of maternal heterochrony, the reproductive ground plan, and the genetic toolkit hypothesis. Finally, the role of environmental and epigenetic factors is discussed from an eco-evo-devo perspective. With this, the authors remind us to keep an eye on incipiently and facultatively social bees, if the concern is to understand the biology underlying the origins of insect sociality.

The third article, provided by Angel Roberto Barchuk and coworkers, reviews and provides new data concerning the question of castespecific development of the honey bee brain. Knowingly, workers are excellent models for neural networks driving complex behaviors, but how is their brain built, and do they differ from queens in this respect? The authors review data on the growth dynamics of the brain in queen and worker larvae and pupae, and they provide insights on the expression patterns of five genes considered important for the differential development of the mushroom bodies. Furthermore, an interesting new data set provides evidence for left-right structural asymmetry of the brain hemispheres and how this arises in the worker brain during pupal development.

The fourth article, coming from my group, is a review on gene expression patterns related to the development of the honey bee ovary. Starting with 
the embryonic stage, where very little is known yet about how the reproductive system of honey bees is actually set up, the main focus is then set on differential gene expression in the larval ovary, particularly on the control of programmed cell death in the worker ovary. Programmed cell death is also an important facet of the repressed ovarian activity in adult queenright workers. Finally, the very high ovariole number of honey bee queens, compared to all other bees, is discussed in the context of the idiosyncratic reproductive biology of the genus Apis, and this leads to the question as to why not only queens but also drones also may have developed exaggerated gonad morphologies.

The last, fifth, article reports on clock genes that drive the circadian rhythm of adult worker honey bees. The 2017 Nobel Prize in Physiology or Medicine has been awarded to three researchers who revealed the molecular organization and machinery underlying the circadian rhythm in animals, so the current report by Fabiano Abreu and colleagues is a most timely contribution on this issue in honey bees. They report on the daily expression profile of eight clock genes in adult workers of different chronological age. Furthermore, the authors propose a microRNA-mRNA regulatory network for two of the principal clock genes. These results are of interest because adult honey bee workers are considered arrhythmic while performing brood care tasks, but exhibit a perfect daily activity rhythm once they become foragers. 